\title{
An Innovation in Suction Assisted FUE
}

\section{Roberto Trivellini, MD Asunción, Paraguay Irtrivellini@yahoo.com.ar}

${ }^{*}$ Disclosure: Dr Trivellini is the owner of Mamba Instruments, which is the manufacturer of the Mega FUE ${ }^{\circledR}$ device described in this article.

\section{Introduction}

We know that the angle of the hair changes as it emerges from the scalp because we are able to see it; it is tangible. However, what is beneath the epidermis is usually a puzzle for the FUE surgeon. In the three-dimensionality of a follicular unit, each hair usually has a different path from the bulb until its departure from the skin. The surgeon can only estimate this, since it is not visible. Axial movement applied to the punch by the surgeon's hand during insertion undergoes changes of direction in its path as it penetrates the skin. This causes constant modification of the resulting force vectors in the dynamics of follicle extraction. Since the punch movement is unpredictable, its path and its geometrical shape are very irregular. Nevertheless, the surgeon's hand movement is much better than the linear motion of the punch performed by a robotic arm. Given these realities, the advance of a punch blindly into the scalp almost becomes a game of chance in which there can only be a loser, the patient.

We have developed a new device and technique for FUE that 1) improves the margin of safety in the extraction of the follicular unit, 2) provides better control of the punch movements into the scalp, and 3) facilitates safe removal of the follicular unit.

Links to the following videos have been included: Video 1: Positioning of the punch prior to insertion; Video 2: Follicular unit buckling; Video 3: Effect of sonic vibration on tissue.

\section{The Device}

The device consists of an HMI (Human Machine Interface) or control panel (Figure 1), handpieces (Figure 2), and a suction unit (Figure 3). The HMI is a touch screen, with independent controls for two handpieces. Three types of punch movement can be programmedrotation, oscillation, and vibration-that can be set to run independently or in a sequence. The handpieces are straight with attached suction. They are lightweight and ergonomic. They can be autoclaved. The rotary Tyler chuck allows for insertion of punches of different sizes, and the nose cone controls punch depth. The suction unit is a device with an oscillating piston, which has a capacity to generate $600 \mathrm{mmHg}$ of negative pressure. The most important feature is the suction pump aspiration capacity, which is affected by the diameter and length of the tubing and pressure losses in the circuit. The volume aspirated in by the punch is $200 \mathrm{cc} \mathrm{H}_{2} \mathrm{O} /$ min with a $1.0 \mathrm{~mm}$ punch and $150 \mathrm{cc} \mathrm{H}_{2} \mathrm{O} /$ min with a $0.8 \mathrm{~mm}$ punch. We have just completed our most recent design of the device, which incorporates the suction unit into the body of the control panel (Figure 4).

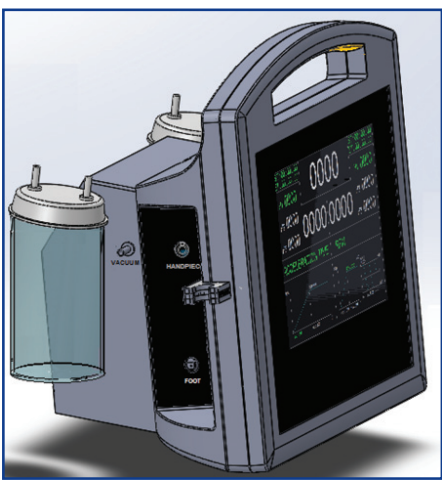

Figure 4. Newest model with suction incorporated

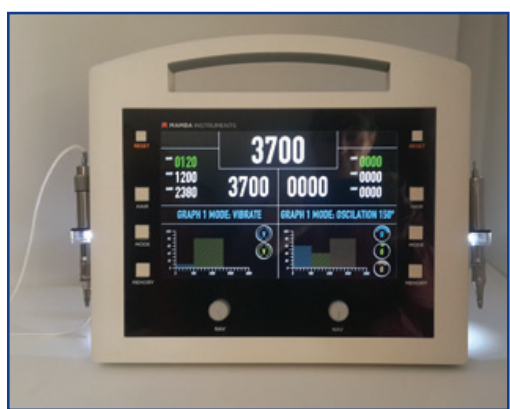

Figure 1. Mega FUE control panel

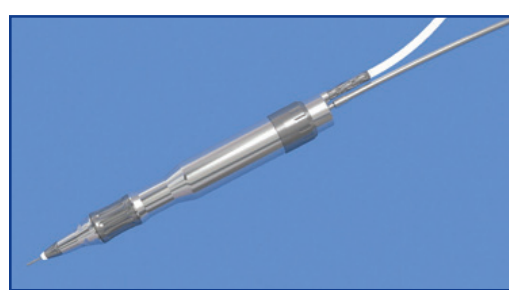

Figure 2. Mega FUE handpiece with suction

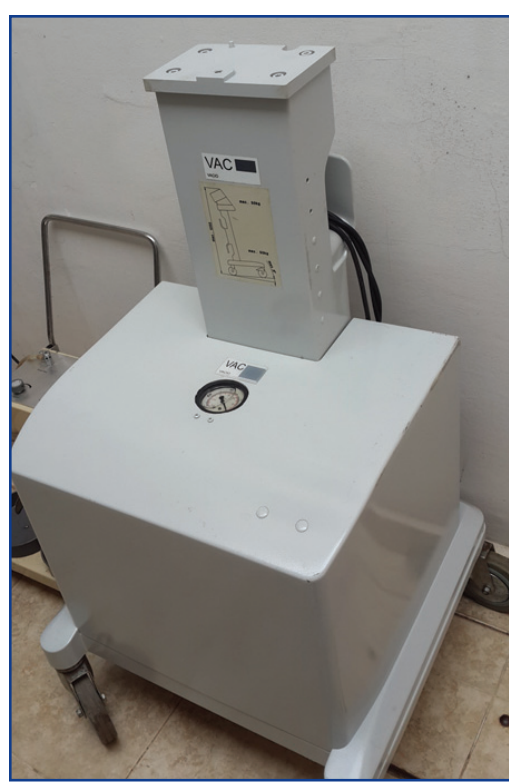

Figure 3. Suction unit (liposuction type)
I use my own sharp punches made of martensitic stainless steel, 17-4 PH, a material that holds its edge allowing the punch to work properly for a longer period. The punches have an inner bevel and outer edge and external diameters vary between 0.70 and $1 \mathrm{~mm}$. I prefer a $0.88 \mathrm{~mm}( \pm 0.002)$ punch for scalp harvesting as this diameter punch, with the cutting edge on the outer surface, paired with suction allows me to extract units of 1-4 intact hairs with minimal transection.

\section{Dynamics of the Technique}

The punch can be maneuvered upon first contact with the surface of the skin into the correct position of insertion (Video 1: https://vimeo. com/154235244). Once in position, the suction of the handpiece applies enough pressure that the punch remains fixed in position (Figure 5). This fixation prevents lateral movement of the punch when it starts rotating. This happens with traditional rotary motors when the punch has

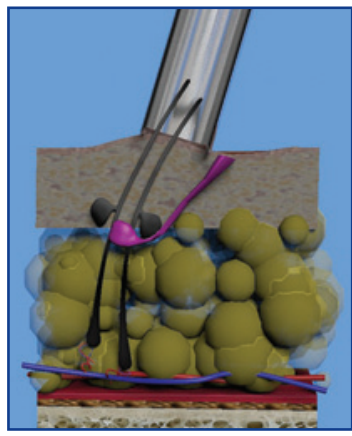

Figure 5. Punch fixed in position by suction lost its edge or when contact is at the wrong angle with the punch turning at high speed.

With the punch held in position by the suction, we apply enough pressure to depress the skin surface about $2 \mathrm{~mm}$. At this point, there is a unidirectional axial load on the follicular unit and a force of equal magnitude in the opposite direction is generated outward from the skull. This causes a lateral deformity or bending of the less dense tissue in the pathway of the punch. The follicles in the follicular unit exhibit a phenomenon called elastic buckling instability (Figure 6). The punch begins rotating only after depressing the skin $2 \mathrm{~mm}$. The speed and degree of punch rotation is set on the control pad. A foot pedal starts and stops punch rotation. As the punch begins to rotate, it is initially held stationary in place and not advanced. 
Elasticity is the property of certain materials to be deformed when they are subjected to the action of external forces and to recover the original shape when these forces are removed (i.e., a reversible deformity). Skin has this elastic property (Figure 7). As the punch starts rotation, the upper layers of the skin (which have the greatest rigidity or cohesion) are cut. The less cohesive tissue located below the depth of the cutting edge of the punch is relieved of the axial load. The follicles of the unit being extracted, which are charged with potential energy due to their compression, recoil, seeking to recover original form (Video 2: https:// vimeo.com/154235465).

They rise up entering into the rotating punch as it is held at the same depth.

The addition of suction to the punch adds force

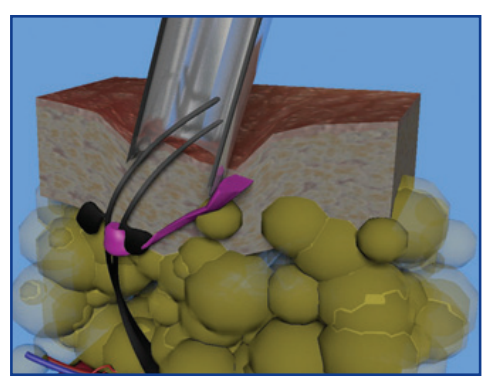

Figure 6. Follicular unit buckling due to axial force

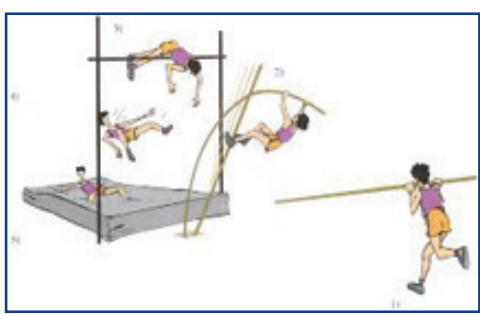

Figure 7. An illustration of elastic force

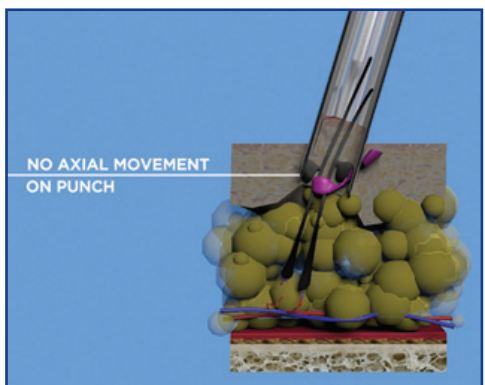

Figure 8. Deep punch penetration without rotation that pulls the excised tissue even further into the punch lumen. Rotation pauses for a split second after the dissection of the graft tissue to level of the sebaceous gland is complete and the graft has entered into the punch (Figure 8).

The need to stop rotation at this point is understandable as follows. When using a punch with an outer cutting edge, the friction of the tissue on the inner wall of the punch is greater than on the outer wall, and at the level of the sebaceous gland, friction is even higher. The result is increased drag and torque of the follicles in the graft. In addition, if the punch is $0.9 \mathrm{~mm}$ external diameter and 0.7 internal diameter, the $0.9 \mathrm{~mm}$ graft obtained will be compressed to $0.7 \mathrm{~mm}$ as it enters the punch. This compression increases the graft tissue density and rigidity making it more vulnerable to pressure, torsional forces, and damage during punch rotation. This explains why most surgeons limit the insertion depth of sharp rotating punches to this level.

Once the punch has stopped rotation and the graft is separated to the level of the sebaceous gland, the punch begins to vibrate. Vibration is the reciprocating movement of a machine or element in any spatial direction from its equilibrium position. Energy waves disseminate through the adjacent tissue when the punch vibrates. This causes a rhythmic motion of tissue, which results in alternating compression and expansion (Video 3: https:// vimeo.com/154235736). This micro-movement rhythmically separates the surface of the graft tissue in contact with the punch and facilitates the drawing action of the suction.

Suction applied at this time is very effective because tissue density at this level of the dermis is low. It is adipose tissue consisting essentially of triglycerides, has a semi-liquid state with a density of about $0.9 \mathrm{~g} / \mathrm{ml}[0.9 \mathrm{~kg} / \mathrm{L}]$, and if we use additional fluid tumescence, we further reduce the tissue density by increasing the volume of the tissue.

The punch suction produces traction on the graft, stretching it and facilitating penetration of the punch. For the first time since I started performing FUE, because of the effect of the suction, I am able to move deeper with the punch to the level of the bulbs without increasing risk of transection. During this deeper insertion, the punch doesn't rotate or oscillate in performing this additional punch penetration, I use the tip of my middle finger as a pivot and push the punch deeper in a fast motion, like the strike attack by a mamba snake-hence the name I give to this technique. The movement must be rapid to prevent complete removal of the graft by the suction. Our goal is to have the graft be virtually free but still connected to its blood supply while awaiting extraction. If the punch passes the level of the bulbs, we would essentially be doing a full excision or a micro liposuction of the unit. In order to set the depth limit for the punch insertion, we measure test grafts at the beginning of the procedure and adjust the tip of the handpiece to the desired depth.

\section{Conclusion}

We have developed a new programmable motorized FUE device with features not present in previously developed devices. The device is unique in how it uses suction and in the variety of sequences of punch movement that it employs. In our hands, the device has improved graft quality, reduced transections, and made it easier to remove the grafts without trauma.

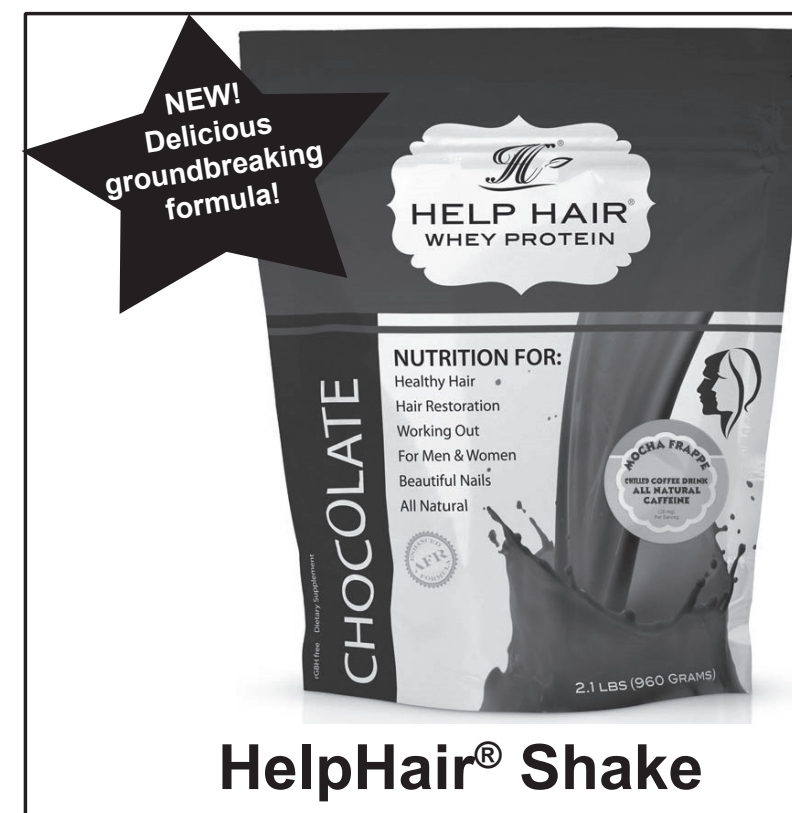

$$
\begin{gathered}
\text { Mocha Frappe } \\
\text { Delicious for your hair }{ }^{\mathrm{TM}}
\end{gathered}
$$

Call today for free samples, SHAPIRO Pocket Chart and wholesale prices!

We fully train your staff.

Join our Worldwide HelpHair ${ }^{\circledR}$ Clinics today! info@Helphair.com•1-561-499-2111 